\title{
Cultura de segurança do paciente em emergências pediátricas
}

\section{Culture of patient safety in pediatric emergencies}

\section{Cultura de seguridade del paciente en emergencias pediátricas}

\author{
Taise Rocha Macedo ${ }^{1}$, Patrícia Kuerten Rocha² , Carla Susana Martinez Julca ${ }^{3}$, Luciane Possoli
}

Informações do Artigo:

Recebido em: 01/07/2019

Aceito em: 24/08/2020

\begin{abstract}
DOI:
RESUMO

Objetivo: avaliar as variáveis percepção geral e nota de segurança do paciente, bem como, frequência e número de eventos comunicados do instrumento Hospital Survey on Patient Safety Culture na perspectiva da equipe de enfermagem em Emergências Pediátricas. Metodologia: Pesquisa quantitativa, tipo survey transversal, amostra intencional, não probabilística, participando do estudo 75 profissionais da equipe de enfermagem. Os dados foram submetidos à análise descritiva e estatística com aplicação do teste Qui-Quadrado. Resultados: Análise da variável de resultado não identificou diferença significativa entre as respostas das categorias profissionais. $\mathrm{Na}$ dimensão percepção geral da segurança do paciente os auxiliares em enfermagem atribuíram maior percentual de respostas positivas dentro das categorias profissionais, enquanto os enfermeiros conferiram a maior proporção de respostas negativas. 0 número de eventos adversos comunicados foi pouco expressivo. Conclusão: $A$ categoria profissional pode interferir na avaliação da cultura de segurança e a cultura de segurança não está completamente instituída nestes serviços.
\end{abstract}

DESCRITORES:

Segurança do paciente; Cultura organizacional; Enfermagem pediátrica.

\section{ABSTRACT}

Objective: to evaluate the variables general perception and patient safety score, as well as, frequency and number of events reported by the Hospital Survey on Patient Safety Culture from the perspective of the nursing team in Pediatric Emergencies. Methodology: Quantitative research, type cross-sectional survey, intentional sample, not probabilistic, participating in the study 75 professionals of the nursing team. The data were submitted to descriptive and statistical analysis using the Chi-Square test.

Results: Analysis of the result variable did not identify a significant difference between

1 Universidade Federal de Santa Catarina. Endereço: Rua José de Souza Machado, SN- Centro, Maracajá- SC. (48) 988324654.E-mail: taiserm16@gmail.com.

Universidade Federal de Santa Catarina. E-mail: pkrochaucip@gmail.com.

3Universidade Federal de Santa Catarina. E-mail: carla.martinez@upch.pe.

4Universidade Federal de Santa Catarina. E-mail: lupossolidb@gmail.com. 


\begin{abstract}
the responses of the professional categories. In the dimension of general perception of patient safety, nursing assistants attributed a higher percentage of positive responses within the professional categories, while nurses conferred the highest proportion of negative responses. The number of adverse events reported was insignificant. Conclusion: The professional category can interfere in the evaluation of the safety culture and the safety culture is not fully established in these services.

DESCRIPTORS:

Patient safety; Organizational culture; Pediatric nursing.

\section{RESUMEN}

Objetivo: evaluar las variables percepción general y puntaje de seguridad del paciente, así como la frecuencia y el número de eventos reportados por la Encuesta del Hospital sobre Cultura de Seguridad del Paciente desde la perspectiva del equipo de enfermería en emergencias pediátricas. Metodología: Investigación cuantitativa, encuesta transversal, muestra intencional, no probabilística, participando en el estudio 75 profesionales del equipo de enfermería. Los datos se sometieron a un análisis descriptivo y estadístico utilizando la prueba de Chi-Square. Resultados: El análisis de la variable de resultado no identificó una diferencia significativa entre las respuestas de las categorías profesionales. En la dimensión de la percepción general de la seguridad del paciente, los asistentes de enfermería atribuyeron un mayor porcentaje de respuestas positivas dentro de las categorías profesionales, mientras que las enfermeras otorgaron la mayor proporción de respuestas negativas. El número de eventos adversos informados fue insignificante. Conclusión: la categoría profesional puede interferir en la evaluación de la cultura de seguridad y la cultura de seguridad no está completamente establecida en estos servicios.
\end{abstract}

DESCRIPTORES:

Seguridad del paciente; Cultura de la organización; Enfermería pediátrica.

\title{
INTRODUÇÃO
}

A segurança do paciente é tema de preocupação desde os primórdios da Medicina, quando há mais de dois mil anos Hipócrates já falava em não causar dano ao paciente. Na área da Enfermagem, Florence Nightingale afirmava que o primeiro dever de um hospital deveria ser não causar mal ao paciente $^{(1-2)}$.

No entanto, foi a partir da publicação do relatório To Err is Human: Building a Safer Health Care System, no ano de 1999 que a segurança do paciente se tornou prioridade fundamental para os serviços de saúde, quando este estudo revelou que cerca de 44.000 a 98.000 pessoas morriam anualmente nos Estados Unidos em decorrência dos erros médicos, sendo que atualmente as estimativas anuais são de 400.000 mortes $^{(3-4)}$.

Na população pediátrica a situação é ainda mais complexa, quando estudos apontam que a ocorrência de eventos adversos com risco de dano nesta população, é três vezes maior quando comparados a adultos submetidos à mesma situação(5).

Diante destas circunstâncias, melhorar a segurança do paciente deve ser um princípio fundamental na assistência à saúde, principalmente nos locais de atendimento especializados como as emergências pediátricas(6).

Afinal, estas unidades são locais propensos a ocorrência de erros e eventos adversos, pois 
trabalham com as especificidades do público pediátrico, quantitativo reduzidos de profissionais, grande demanda de atendimento e vivenciam constantemente situações de risco a vida(7).

Apesar da necessidade de fortalecer a cultura de segurança neste contexto para modificarmos 0 atual cenário da segurança do paciente, percebe-se que os estudos envolvendo a temática ainda são incipientes. Neste sentido, este estudo traz como objetivo avaliar as variáveis percepção geral e nota de segurança do paciente, bem como, a frequência e número de eventos comunicados do instrumento Hospital Survey on Patient Safety Culture na perspectiva da equipe de enfermagem.

\section{METODOLOGIA}

Trata-se de um estudo descritivo exploratório, de cunho quantitativo, do tipo survey transversal, realizado em três Unidades de Emergência Pediátrica, localizadas em Florianópolis, Santa Catarina, Brasil.

A população foi composta pela equipe de enfermagem das três Emergências Pediátricas, totalizando 91 profissionais. A amostra foi intencional, não probabilística, seguindo os seguintes critérios de inclusão: ser enfermeiro, técnico ou auxiliar em enfermagem e estar no exercício de suas funções nas emergências pediátricas no período da coleta de dados; profissionais admitidos e lotados no setor há mais de dois meses (devido ao período de adaptação necessário, podendo o funcionário não ter total conhecimento acerca da unidade). Como critérios de exclusão consideraram-se: profissionais da enfermagem em férias, licença saúde, licença gestação, licença prêmio durante o período da coleta de dados; profissionais da enfermagem com menos de dois meses de trabalho na unidade ou que não estejam lotados na referida unidade; instrumentos preenchidos em menos da metade; e/ou desistência do consentimento após início da coleta de dados.

Seguindo tais critérios, 77 profissionais estavam aptos a participar do estudo. No entanto, dois indivíduos não manifestaram o aceite, compondo a amostra final 75 participantes.

Realizou-se a coleta de dados entre setembro e novembro de 2014 por meio da aplicação do instrumento HSOPSC validado para o contexto hospitalar brasileiro(8), após a autorização dos autores originais $(9)$.

O instrumento foi elaborado pela Agency for HealthcareResearchandQuality (AHRQ), e possui 42 itens relacionados à cultura de segurança do paciente. Buscando garantir respostas mais consistentes, 18 itens do instrumento são reversos, ou seja, nestes casos quando o participante discorda do item formulado negativamente estará expressando sua opinião de forma positiva(9).

Estes itens estão dispostos em três grupos de variáveis: variáveis de características profissionais do sujeito; variáveis da cultura de segurança e variáveis de resultado. Neste estudo optou-se por avaliar as variáveis de resultado, buscando explorar com maior profundidade os itens que a compõem. As 
variáveis de resultado, são composta por nove itens do instrumento: Percepção geral da segurança do paciente, formada pelos itens A10, A15, A17 e A18; Frequência de eventos comunicados, formada pelos itens D1, D2, D3; Número de eventos comunicados, item $\mathrm{G}$ e Nota de segurança do paciente, item $F^{(9)}$.

0 início da coleta de dados se deu mediante a autorização das chefias de enfermagem das unidades. Posteriormente, o estudo foi apresentado individualmente para cada participante de acordo com sua disponibilidade de tempo na unidade. Após os indivíduos manifestarem seu aceite em participar da pesquisa através da assinatura do Termo de Consentimento Livre e Esclarecido foi entregue um envelope opaco contento o instrumento de coleta de dados; após os mesmos responderem o instrumento os inseriram novamente no envelope e de forma anônima os depositavam em uma urna disponível na unidade. Ao final de cada período, os envelopes já preenchidos e inseridos na urna eram recolhidos pela pesquisadora.

Para a análise dos dados inicialmente todas as respostas obtidas foram tabuladas em uma planilha do Microsoft Excel. Nas análises descritivas, as respostas foram recodificadas em positivas quando no instrumento continham as seguintes respostas: concordo totalmente, concordo, sempre e quase sempre; negativas: discordo totalmente, discordo, nunca e raramente; e neutras: não concordo nem discordo e às vezes.

Para analisar se houve diferença estatística nas respostas da variável de resultado entre as diferentes categorias profissionais e entre as respostas apresentadas dentro da própria categoria, utilizou-se o programa $R 3.1 .1$ com base no pacote stats $\left({ }^{(10)}\right.$, adotando nível de significância de $p \leq 0,05$ utilizando o teste Qui-Quadrado.

As questões éticas seguiram as recomendações da Resolução 466/2012 do Conselho Nacional de Saúde, sendo submetido e aprovado na Plataforma Brasil sob o protocolo 35231514.1.0000.5361(11).

\section{RESULTADOS}

Participaram do estudo 75 profissionais de enfermagem, sendo estes predominantemente técnicos em enfermagem 40 (53\%), auxiliares em enfermagem 18 (24\%) e enfermeiros 17 (23\%). Destes, 14 (19\%) possuíam de um a cinco anos de trabalho na profissão, 18 (24\%) de 6 a 10 anos, seis (8\%) de 11 a 15 anos e oito (11\%) entre 16 e 20 anos.

Para identificar como estas diferentes categorias profissionais avaliaram a variável de resultado do instrumento HSOPSC, procedeu-se a análise de cada grupo de itens do instrumento.

A variável, Percepção Geral da Segurança do Paciente permite avaliar se os procedimentos e sistemas são adequados para prevenir erros, enganos e falhas promovendo a segurança do paciente ${ }^{(9)}$.

Assim, analisando os resultados apresentados na mesma com o intuito de identificar possíveis diferenças estatísticas entre as respostas apresentadas pelas diferentes categorias profissionais que 
participaram do estudo, pode-se observar que não se identificou diferença significativa quando os valores foram agrupados em respostas positivas, neutras e negativas entre 0 conjunto geral de resposta $\left(x^{2}=\right.$ $0,12 ; p=0,99)$ e entre os cargos, conforme tabela 1 .

Tabela 1. Comparação de respostas positivas, neutras e negativas dos itens que estruturam a dimensão Percepção Geral da Segurança do Paciente de acordo com as categorias profissionais da equipe de enfermagem de emergência pediátrica de hospitais públicos da Grande Florianópolis, Santa Catarina, Brasil, 2014

\begin{tabular}{|c|c|c|c|c|}
\hline \multicolumn{5}{|c|}{ A10- É apenas por acaso, que erros, enganos ou falhas mais graves não acontecem neste setor } \\
\hline Categoria Profissional & Positivo n (\%) & Neutro n (\%) & Negativo $n(\%)$ & $\mathrm{p}$ \\
\hline Enfermeiro & $10(59)$ & $02(12)$ & $04(24)$ & 0,07 \\
\hline Técnico em Enfermagem & $20(50)$ & $05(13)$ & $15(37)$ & 0,18 \\
\hline Auxiliar em Enfermagem & $10(55)$ & $03(17)$ & $05(28)$ & 0,63 \\
\hline \multicolumn{5}{|c|}{ A15- A segurança do paciente jamais é comprometida em função da maior quantidade de trabalho a } \\
\hline Categoria Profissional & Positivo $n(\%)$ & Neutro n (\%) & Negativo n (\%) & $p$ \\
\hline Enfermeiro & $2(12)$ & $2(12)$ & $13(76)$ & $<0,01$ \\
\hline Técnico em Enfermagem & $15(38)$ & $7(17)$ & $18(45)$ & 0,56 \\
\hline Auxiliar em Enfermagem & $10(56)$ & $2(11)$ & $6(33)$ & 0,38 \\
\hline \multicolumn{5}{|c|}{ A17- Nesta unidade temos problemas de segurança do paciente ${ }^{*}$} \\
\hline Categoria Profissional & Positivo n (\%) & Neutro n (\%) & Negativo $\mathrm{n}(\%)$ & $p$ \\
\hline Enfermeiro & $1(6)$ & $1(6$ & $15(88)$ & $<0,01$ \\
\hline Técnico em Enfermagem & $9(22)$ & $4(10)$ & $27(68)$ & $<0,01$ \\
\hline Auxiliar em Enfermagem & $7(39)$ & $7(39)$ & $4(22)$ & 0,17 \\
\hline \multicolumn{5}{|c|}{ A18- Os nossos procedimentos são adequados para prevenir a ocorrência de erros* } \\
\hline Categoria Profissional & Positivo n (\%) & Neutro n (\%) & Negativo n (\%) & $p$ \\
\hline Enfermeiro & $1(6)$ & $6(35)$ & $10(59)$ & $<0,01$ \\
\hline Técnico em Enfermagem & $15(38)$ & $5(12)$ & $20(50)$ & $<0,01$ \\
\hline Auxiliar em Enfermagem & $10(56)$ & $3(17)$ & $5(27)$ & 0,14 \\
\hline
\end{tabular}

Percepção Geral da Segurança do Paciente*

\begin{tabular}{ccccccc}
\hline Categoria Profissional & Positivo n (\%) & $p$ & Neutro n (\%) & P & Negativo n (\%) & p \\
Enfermeiro & $14(21)$ & & $11(16)$ & & $42(63)$ & \\
\hline
\end{tabular}


Técnico em Enfermagem

$59(17)$

$21(13)$

$80(50)$

Auxiliar em Enfermagem

$37(51)$

0,9

$15(21)$

0,9

$20(28)$

*Teste Qui-Quadrado

Fonte: Elaborado pelas autoras, 2019.

Na análise geral da variável Percepção Geral da Segurança do Paciente, a possibilidade de respostas para cada categoria profissional são: 67 (100\%) para os enfermeiros, 160 (100\%) para os técnicos em enfermagem e 72 (100\%) para os auxiliares em enfermagem, de acordo com o quantitativo de participantes de cada categoria profissional e a quantidade de itens que compõe a variável.

Neste sentido, nesta variável a categoria profissional que apresentou o maior índice de resposta positiva foram os auxiliares em enfermagem com $37(51 \%)$ das respostas, e de repostas negativas foram os enfermeiros com 42 (63\%). Na avaliação das respostas neutras, a proporção destas respostas não oscilou demasiadamente de acordo com a categoria profissional, conforme demonstrado na tabela 1.

No entanto, particularidades foram encontradas na análise de cada item. No item A15 "A segurança do paciente jamais é comprometida em função da maior quantidade de trabalho a ser concluída" quando foram analisadas as diferenças entre as respostas positivas, neutras e negativas dentro de cada cargo, houve diferença estatística entre as respostas para o cargo de enfermeiro $\left(x^{2}=13,88 ; p<0,01\right)$, exibidos na tabela 1.

Já no item A17 "Nesta unidade temos problemas de segurança do paciente" quando foram analisados os valores obtidos dentro de cada cargo, inicialmente houve diferença significativa para 0 cargo de enfermeiro $\left(x^{2}=23,89 ; p<0,01\right)$ e para o cargo de técnico em enfermagem $\left(x^{2}=32,25 ; p<0,01\right)$, evidenciados na tabela 1.

E no item A18 "Os nossos procedimentos e sistemas são adequado para prevenir a ocorrência de erros", quando as respostas foram avaliadas dentro de cada cargo, no geral, não houve diferença significativa $\left(x^{2}=15,32 ; p=0,053\right)$, cuja variação manteve-se significativa para as respostas dentro do cargo enfermeiro $\left(x^{2}=18,0, p<0,01\right)$ e cargo técnico em enfermagem $\left(x^{2}=13,5 ; p<0,01\right)$, conforme apresentado na tabela1.

A segunda variável avaliada foi "Frequência de Eventos Comunicados", que é composta por três itens do instrumento original (D1, D2 e D3), sendo que estes itens permitem identificar se os erros são comunicados, detectados e corrigidos antes de prejudicar o paciente, independentemente de serem erros com ou sem potencial de prejudicá-lo, conforme tabela 2.

Tabela 2. Frequência de eventos adversos comunicados de acordo com as categorias profissionais da equipe de enfermagem de emergência pediátrica de hospitais públicos da 
Grande Florianópolis, Santa Catarina, Brasil, 2014

D1- Quando ocorre erro, engano ou falha, mas ele é percebido e corrigido antes de afetar o paciente, com que frequência ele é relatado?*

\begin{tabular}{|c|c|c|c|c|}
\hline Categoria Profissional & Positivo n (\%) & Neutro n (\%) & Negativo $\mathrm{n}(\%)$ & $p$ \\
\hline Enfermeiro & $11(65)$ & $1(6)$ & $5(29)$ & 0,04 \\
\hline Técnico em Enfermagem & $13(33)$ & $9(22)$ & $18(45)$ & 0,17 \\
\hline Auxiliar em Enfermagem & $11(61)$ & $1(6)$ & $4(22)$ & 0,1 \\
\hline \multicolumn{5}{|c|}{$\begin{array}{l}\text { D2- Quando ocorre erro, engano ou falha, mas não há risco de dano ao paciente, com que frequênci } \\
\text { ele é relatado?* }\end{array}$} \\
\hline Categoria Profissional & Positivo $\mathrm{n}(\%)$ & Neutro n (\%) & Negativo n (\%) & $p$ \\
\hline Enfermeiro & $12(70$ & $3(18)$ & $2(12)$ & $<0,01$ \\
\hline Técnico em Enfermagem & $10(25)$ & $13(32)$ & $17(43)$ & 0,03 \\
\hline Auxiliar em Enfermagem & $11(61)$ & $4(22)$ & $2(11)$ & 0,09 \\
\hline \multicolumn{5}{|c|}{ D3- Quando ocorre erro, engano ou falha que poderia causar danos ao paciente, mas não causa co } \\
\hline Categoria Profissional & Positivo n (\%) & Neutro n (\%) & Negativo $n(\%)$ & $p$ \\
\hline Enfermeiro & $11(69)$ & $3(19)$ & $2(12)$ & 0,07 \\
\hline Técnico em Enfermagem & $12(30)$ & $17(43)$ & $11(27)$ & 0,01 \\
\hline Auxiliar em Enfermagem & $14(78)$ & $1(6)$ & $2(11)$ & 0,01 \\
\hline
\end{tabular}

Frequência de eventos adversos comunicados em Unidades de Emergência Pediátrica *

\begin{tabular}{ccccccc}
\hline Categoria Profissional & Positivo $n(\%)$ & $p$ & Neutro $n(\%)$ & $P$ & Negativo $n(\%)$ & $p$ \\
Enfermeiro & $34(68)$ & & $7(14)$ & & $9(18)$ & \\
Técnico em Enfermagem & $35(29)$ & 0,96 & $39(33)$ & 0,9 & $46(38)$ & 0,91 \\
Auxiliar em Enfermagem & $36(72)$ & & $6(12)$ & 1 & $8(16)$ & \\
\hline
\end{tabular}

*Teste Qui-Quadrado

Fonte: Elaborado pelas autoras, 2019.

Na variável Frequência de eventos adversos comunicados, a possibilidade de respostas para cada categoria profissional são: 50(100\%) para os enfermeiros, 120 (100\%) para os técnicos em enfermagem e $50(100 \%)$ para os auxiliares em enfermagem, de acordo com o quantitativo de participante de cada categoria profissional e a quantidade de itens que compõe a variável.

A tabela 2 aponta que a categoria profissional que apresentou maior frequência de respostas positivas nesta variável foram os auxiliares em enfermagem com 36 (72\%) respostas, negativas foram os enfermeiros 34 (68\%) e neutra também foram os técnicos em enfermagem com 39 (33\%) respostas. 
Mas, apesar destas discrepâncias, na análise geral da dimensão não foi possível identificar diferença estatística significativa quando agrupadas as respostas em positivas, neutras e negativas $\left(x^{2}=0,46\right.$; $p=0,98)$, da mesma forma quando se comparou as diferenças entre as categorias profissionais.

Quando os itens foram avaliados individualmente surgiram algumas especificidades. Na análise estatística do primeiro item desta variável - D1 "Quando ocorre erro, engano ou falha, mas ele é percebido e corrigido antes de afetar o paciente, com que frequência ele é relatado?", observou-se que apenas os enfermeiros apresentaram diferença estatística significativa quando avaliado $\left(x^{2}=9,77 ; p=0,04\right)$ como mostra a tabela 2.

Ainda na tabela 2 no item D2 "Quando ocorre erro, engano ou falha, mas há risco de dano ao paciente, com que frequência ele é relatado?", identificou-se diferença estatisticamente significativa ao analisar individualmente os enfermeiros $\left(x^{2}=13,19 ; p<0,01\right)$ e os técnicos em enfermagem $\left(x^{2}=10,75\right.$; $p=0,03)$

E, no último item desta variável, também apresentado na tabela 2 - D3 "Quando ocorre erro, engano ou falha que poderia causar danos ao paciente, mas não causa, com que frequência ele é relatado?", também houve diferença quando avaliadas individualmente os técnicos em enfermagem $\left(x^{2}=16,0 ; p<0,01\right)$ e auxiliares em enfermagem $\left(x^{2}=13,88 ; p<0,01\right)$.

A terceira variável avaliada foi "Nota de segurança do paciente", e permite que através do instrumento os participantes atribuam uma nota de segurança do paciente dentro de cinco possibilidades: excelente, muito bom, regular, ruim e muito ruim. Analisando as notas obtidas, observou-se que os auxiliares em enfermagem foram aqueles que avaliaram a segurança do paciente mais positivamente 12 $(66 \%)$, enquanto os enfermeiros foram os que mais avaliaram negativamente $4(24 \%)$, conforme se visualiza na tabela 3 .

Tabela 3. Nota de segurança do paciente e número de eventos adversos comunicados pelas categorias profissionais da equipe de enfermagem das emergências pediátricas de hospitais públicos da Grande Florianópolis, Santa Catarina, Brasil, 2014 


\begin{tabular}{ccccccc}
\hline \multicolumn{7}{c}{ Nota de Segurança do Paciente * } \\
\hline Categoria & Excelente & Muito Bom & Regular & Ruim & Muito Ruim & $\mathrm{p}$ \\
Profissional & $\mathrm{n}(\%)$ & $\mathrm{n}(\%)$ & $\mathrm{n}(\%)$ & $\mathrm{n}(\%)$ & $\mathrm{n}(\%)$ & \\
Enfermeiro & - & $5(29)$ & $8(47)$ & $3(18)$ & $1(6)$ & 0,02 \\
Téc. em Enferm. & $2(5)$ & $13(33)$ & $17(43)$ & $5(13)$ & $2(5)$ & 0,01 \\
Aux. em Enferm. & $4(22)$ & $8(44)$ & $3(17)$ & - & - & 0,01 \\
\hline Categoria & Nenhum & 1 a 2 & 3 a 5 & 6 a 10 & 11 a 20 & $\mathrm{p}$ \\
Profissional & $\mathrm{n}(\%)$ & $\mathrm{n}(\%)$ & $\mathrm{n}(\%)$ & $\mathrm{n}(\%)$ & $\mathrm{n}(\%)$ & \\
Enfermeiro & $4(24)$ & $8(47)$ & $5(29)$ & - & - & 0,01 \\
Téc. em Enferm. & $19(48)$ & $13(32)$ & $6(15)$ & - & $2(5)$ & 0,01 \\
Aux. em Enferm. & $11(61)$ & $5(28)$ & $1(6)$ & $1(6)$ & - & 0,01 \\
\hline
\end{tabular}

*Teste Qui-Quadrado

Fonte: Elaborado pelas autoras, 2019.

Estatisticamente quando avaliadas as respostas de cada cargo individualizadas, obteve-se diferença significativa de respostas dentro das categorias profissionais. No entanto, a tabela 3 ainda evidencia que quando avaliado as diferenças gerais dentro de cada categoria pesquisada não houve diferença significativa $\left(x^{2}=14,40 ; p=0,07\right)$.

Além de avaliar a nota de segurança do paciente nas instituições, na última variável que compõe a variável de resultado, os participantes apresentaram o quantitativo de eventos adversos relatados nos últimos 12 meses. No geral, os dados apresentados na tabela 3 demonstram que independente da categoria profissional o número de eventos comunicados foi bastante reduzido.

Quando as categorias profissionais foram individualizadas encontraram-se diferenças estatisticamente significativas nas repostas apresentadas, sendo o enfermeiro o profissional que proporcionalmente mais notificou eventos adversos. No entanto, na análise geral entre as categorias profissionais não foi observada diferença estatisticamente significativa $\left(x^{2}=0,61 ; p=0,99\right)$, conforme demonstrado na tabela 3.

\section{DISCUSSÃO}

A cultura de segurança do paciente nas instituições de saúde é estruturada por atitudes, percepções, competências e padrões de comportamento individual ou coletivo dos membros da equipe ${ }^{(12) .}$ Por envolver aspectos individuais, os profissionais podem desenvolver hábitos adequados ou 
não para a segurança do paciente e, por envolver aspectos coletivos, categorias profissionais podem diferir em suas percepções de segurança, sendo neste estudo avaliado apenas os itens que compõem a variável de resultado.

Na primeira dimensão desta variável, "Percepção Geral da Segurança do Paciente", a desproporção de respostas nela encontrada, pode estar relacionada as atribuições executadas pelas diferentes categorias profissionais, dentre as quais pode-se citar que os auxiliares de enfermagem executam atividades de menor complexidade dentro da equipe, e os enfermeiros são responsáveis por aquelas mais complexas que envolvem a direção e organização dos serviços de enfermagem, cuidados de enfermagem que exigem conhecimentos científicos específico e capacidade de tomar decisões imediatas $^{(13)}$.

Outro fator que pode interferir na percepção da segurança do paciente por parte dos profissionais, pode estar relacionado ao processo de formação de cada uma das categorias e ao nível de implantação da cultura de segurança na instituição, fato que estimula a capacidade de relatar, analisar, sistematizar e aprender com os próprios problemas(14).

Aliás, averiguar se os profissionais de saúde identificam problemas de segurança do paciente na instituição é um aspecto relevante para iniciar um processo de transformação destes locais, e está associado ao aperfeiçoamento dos procedimentos, sistemas e consequente prevenção de erros.

Neste sentido, grande proporção dos profissionais que participaram deste estudo não identifica problemas de segurança do paciente em sua unidade, na Arábia Saudita estudo desenvolvido com 2.572 profissionais de dois hospitais, $50 \%$ destes concordaram ou concordaram fortemente que em sua unidade existem problemas de segurança do paciente, sendo uma área potencialmente crítica para a cultura de segurança e potencializadora de mudanças ${ }^{(15)}$.

Estes problemas que envolvem a segurança do paciente podem ser originários dos fluxos de trabalho, procedimentos e sistemas. Deste modo as instituições podem investir e trabalhar nesta tríade a fim de alcançar uma assistência qualificada e segura para os pacientes, prevenindo erros ou relatandoos quando ocorrem procurando buscar adequação(16).

No entanto o que se verificou neste trabalho, é que os enfermeiros e os auxiliares em enfermagem foram os profissionais que comunicaram com maior frequência os eventos adversos, independente dos incidentes causarem ou não danos ao paciente, porém este quantitativo é pouco expressivo quando comparado a outras realidades.

Em um estudo norte americano, a frequência de eventos adversos relatados pelos profissionais, manteve-se consideravelmente mais elevada, $62 \%$ nos incidentes corrigidos antes do dano; $63 \%$ quando os incidentes não tinham potencial de dano e $76 \%$ quando os incidentes poderiam causar dano, mas não causaram; demonstrando que naquele país a cultura de segurança do paciente já está mais bem 
estruturada(9).

Estes resultados reforçam a necessidade de aprimorar a cultura de segurança nas instituições participantes deste estudo, fortalecendo os sistemas de notificação de eventos adversos e estruturando metodologias de aprendizado a partir dos registros obtidos, quando se verifica que independente da categoria profissional o número de eventos adversos comunicados é pequeno e que a diferença estatística entre as categorias não foi significativa.

Esta realidade também foi vivenciada no Líbano, quando $52,7 \%$ dos entrevistados não relataram qualquer evento adverso entre dezembro de 2011 a março de 2012(15) e na Holanda, quando 44,9\% dos profissionais não relataram eventos adversos no período de um ano, sendo que o número de notificações efetuadas pelos enfermeiros foi estatisticamente maior quando comparado à categoria médica(16).

Para alcançar um sistema de notificação eficaz e consequentemente um sistema de saúde mais seguro, é necessário que se reduza os gradientes de hierarquia nas instituições, possibilitando que os indivíduos comuniquem facilmente seus erros, e que estes possam servir de aprendizado, não sendo 0 indivíduo culpabilizado pela sua ocorrência(17).

Apesar das dificuldades de implementação da cultura de segurança, neste estudo se identificou que não houve diferença significativa na nota atribuída à segurança do paciente, quando realizada a análise geral das respostas entre as categorias profissionais. Porém, quando estas foram individualmente analisadas houve diferenças significativas, o técnico em enfermagem foi à categoria profissional que melhor avaliou a segurança do paciente e o enfermeiro a categoria que atribuiu a pior avaliação.

Corroborando com estes achados, estudo brasileiro desenvolvido em quatro Unidades de Terapia Intensiva Neonatal apontou que os técnicos em enfermagem assim como os médicos foram os profissionais que mais atribuíram a nota muito bom para a segurança do paciente de sua unidade. Já os enfermeiros e os auxiliares em enfermagem foram os profissionais que mais atribuíram à nota muito ruim(18).

Os dados deste estudo demonstram que os enfermeiros não estão satisfeitos com o trabalho realizado nas unidades de emergência pediátrica, e que esta insatisfação pode contribuir para a elaboração de estratégias, como a educação permanente, aprimoramento dos processos de comunicação, ajustes nos sistemas de notificação de eventos adversos, substituição da abordagem diante dos erros, fortalecimento do trabalho em equipe, que substituirão o paradigma cultural ainda presente nestas unidades de saúde pela cultura de segurança, que têm como meta central garantir a segurança do paciente.

A cultura de segurança ainda está pouco instituída na área da saúde, assim como ainda é incipiente nos contextos hospitalares. Explorar os aspectos relacionados a avaliação da cultura de 
segurança pelos profissionais de enfermagem é extremamente importante, uma vez que permite identificar áreas potenciais e frágeis da cultura de segurança e que pode refletir em aspectos que devem ser estimulados e trabalhados dentro das instituições.

Este estudo levanta a necessidade de fortalecimento da cultura de segurança dentro das emergências pediátricas, uma vez que as respostas obtidas não apresentam grandes divergências, mesmo que advindas de diferentes categorias profissionais da equipe de enfermagem.

Estimular a ampliação do número de eventos adversos notificados pelos profissionais, permite que as instituições desenvolvam estudos, que podem avançar em mudanças de processos e planos de contingência, refletindo em qualificação profissional e consequente melhora na assistência e segurança do paciente.

Dentre as limitações do estudo, pode-se apontar a participação exclusiva dos profissionais da equipe de enfermagem, deixando os autores a sugestão de replicação deste estudo para a equipe multiprofissional.

\section{CONSIDERAÇÕES FINAIS}

A avaliação da cultura atual é o passo inicial para a implementação de uma cultura de segurança. A categoria profissional pode interferir na avaliação da cultura de segurança e contribuir para a execução de ações que refletirão na sua efetivação.

Os resultados evidenciam que não houve diferença estatisticamente significativa na avaliação das variáveis de resultado apresentadas pelos diferentes profissionais da equipe de enfermagem. Porém, na dimensão percepção geral da segurança do paciente os auxiliares em enfermagem atribuíram o maior percentual de respostas positivas a segurança do paciente quando comparado aos demais membros da equipe.

Reforça-se que o número de eventos adversos comunicados foi pequeno, sendo os enfermeiros os profissionais que proporcionalmente mais notificaram, demonstrando uma fragilidade das unidades participantes do estudo e uma realidade que precisa ser superada.

Ampliar as discussões acerca da cultura de segurança do paciente e melhorar os aspectos que envolvem esta pauta no contexto das Emergências Pediátricas é fundamental para que possamos qualificar os serviços, reduzir o quantitativo de eventos adversos e alcançar instituições de saúde mais seguras.

A partir dos resultados aqui apontados, sugere-se que dentro das suas competências profissionais, a equipe de enfermagem fortaleça a educação permanente, o processo de comunicação, o relato de eventos adversos, o trabalho em equipe dentro das unidades, trazendo à tona a necessidade de substituir a cultura presente nas emergências pediátricas por uma cultura de segurança. 
Novos estudos podem ser desenvolvidos buscando identificar a cultura atual das instituições, para que mudanças sejam implementadas a fim de alcançar um alto nível de segurança do paciente não apenas das Emergências Pediátricas, mas em todo o serviço de saúde.

\section{REFERÊNCIAS}

1. Dickens D, Sinsabaugh D, Winger B. Primum Non Nocere. Arch Pediatric Adolesc Med. 2006; 1620 (11): 1185.

2. Nightingale F. Notes on Hospitals. 3rd ed. London: Longman, Green, Longman, Roberts, and Green; 1863.

3. Konh LT, Corrigan JM, Donaldson MS. Errors in health care: a leading cause of death and injury. In: Konh LT, Corrigan JM, Donaldson MS. To err is human: building a safer health care system. Washington (DC): Institute of Medicine; 2000. p. 26-48.

4. James JA. A new, evidence-based estimate of patient harms associated with hospital care. Journal of Patient Safety. 2013; 9(3): 122-8.

5. Stockwell DC et al. Adverse Events in Hospitalized Pediatric Patients. Pediatrics. 2018; 142(2): e20173360:02-14.

6. Word Health Organization. Patient Safety. [homepage na intenet] 2015. Disponível em: http://www.who.int/topics/patient_safety/en/. Acesso em 18 de fev. de 2015.

7. Mueller BU, Neuspiel DR, Fisher ERS. Principles of Pediatric Patient Safety: Reducing Harm Due to Medical Care. Pediatrics. 2019; 143(2):e20183649.

8. Reis CT, Laguardia J, Vasconcelos AGG, Martins M. Confiabilidade e validade da versão brasileira da Pesquisa Hospitalar sobre Cultura de Segurança do Paciente (HSOPSC): um estudo piloto. Cad. Saúde Pública. 2016; 32(11):e00115614. Disponível em: http://www.scielo.br/scielo.php?script=sci_arttext\&pid=S0102-311X2016001104001\&lng=en. Epub 01 de dezembro de 2016. https://doi.org/10.1590/0102-311x00115614. Acesso: 11 julho de 2020.

9. Famalaro T, Yount N, Hare, R, et al. Hospital Survey on Patient Safety Culture 2018 user comparative database report. (Prepared by Westat, Rockville, MD, under Contract n. HHSA 290201300003C). Rockville, MD: Agency for Healthcare Research and Quality; jun. 2020.

10. R Core Team, R.2018. A language and environment for statistical computing. R Foundation for Statistical Computing. Vienna, Austria; 2018. Disponível em: http://www.R-project.org/ . Acesso em 06 jun 2020.

11. Brasil. Ministério da Saúde. Conselho Nacional de Saúde. Resolução n 466, de 12 de dezembro de 2012. Dispõe sobre diretrizes e normas regulamentadoras de pesquisas envolvendo seres humanos. Disponível em: http://conselho.saude.gov.br/resolucoes/2012/Res0466.pdf. Acesso em: 
06 mai. 2020.

12. Raimondi DC, Bernal SCZ, Oliveira JLC, Matsuda LM. Cultura de segurança do paciente na atenção primária à saúde: análise por categorias profissionais. Rev. Gaúcha Enferm. 2019; 40(spe): e20180133:01-09.

13. Morais Filho LA, et al. Competência legal do enfermeiro na urgência/emergência. Enferm. Foco. 2016; 7(1): 18-23.

14. Costa TD, et al. Percepção de profissionais de enfermagem acerca de segurança do paciente em unidades de terapia intensiva. Rev. Gaúcha Enferm. 2016; 37 (3):1-8.

15. El-Jardali F, Sheikh F, Garcia NA, Jamal D, Abdo A. Patient safety culture in a large teaching hospital in Riyadh: baseline assessment, comparative analysis and opportunities for improvement. BMC Health Services Research. 2014; 14(1): 122.

16. Noord IV, et al. Is culture associated with patient safety in the emergency department? A study of staff perspectives. Int J Qual Health Care. 2013 [citado 2014 Dez 29; (26)6: 573-8. Disponível em: \&lt:http://dx.doi.org/10.1093/intqhc/mzu081\&gt

17. Bernalte-Martía V, Orts-Cortés MI,Maciá-Soler L. Percepción de losprofesionales de enfermería y auxiliares de enfermería sobre cultura de seguridade del paciente enel área quirúrgica. Enfermería clínica. 2015; 25(2): 64-72.

18. Tomazoni A, Rocha PK, Souza SD, Anders JC, Malfussi HFCD. Patient safety culture at neonatal intensive care units: perspectives of the nursing and medical team. Rev. Latino-Am. Enfermagem. 2014; 22(5): 755-63. 